\title{
\begin{tabular}{l|l} 
MitTraries & DSpace@MIT
\end{tabular}
}

\author{
MIT Open Access Articles
}

\section{MAC for Networks with Multipacket Reception Capability and Spatially Distributed Nodes}

The MIT Faculty has made this article openly available. Please share how this access benefits you. Your story matters.

Citation: Celik, G.D. et al. “MAC for Networks with Multipacket Reception Capability and Spatially Distributed Nodes." Mobile Computing, IEEE Transactions on 9.2 (2010): 226-240. (C2011 IEEE.

As Published: http://dx.doi.org/10.1109/tmc.2009.135

Publisher: Institute of Electrical and Electronics Engineers

Persistent URL: http://hdl.handle.net/1721.1/66281

Version: Final published version: final published article, as it appeared in a journal, conference proceedings, or other formally published context

Terms of Use: Article is made available in accordance with the publisher's policy and may be subject to US copyright law. Please refer to the publisher's site for terms of use. 


\title{
MAC for Networks with Multipacket Reception Capability and Spatially Distributed Nodes
}

\author{
Guner D. Celik, Student Member, IEEE, Gil Zussman, Senior Member, IEEE, \\ Wajahat F. Khan, Member, IEEE, and Eytan Modiano, Senior Member, IEEE
}

\begin{abstract}
The physical layer of future wireless networks will be based on novel radio technologies such as UWB and MIMO. One of the important capabilities of such technologies is the ability to capture a few packets simultaneously. This capability has the potential to improve the performance of the MAC layer. However, we show that in networks with spatially distributed nodes, reusing backoff mechanisms originally designed for narrow-band systems (e.g., CSMA/CA) is inefficient. It is well known that when networks with spatially distributed nodes operate with such MAC protocols, the channel may be captured by nodes that are near the destination, leading to unfairness. We show that when the physical layer enables multipacket reception, the negative implications of reusing the legacy protocols include not only such unfairness, but also a significant throughput reduction. We present alternative backoff mechanisms and evaluate their performance via Markovian analysis, approximations, and simulation. We show that our alternative backoff mechanisms can improve both overall throughput and fairness.
\end{abstract}

Index Terms-Multipacket reception, capture, medium access control (MAC), random access, performance evaluation.

\section{INTRODUCTION}

$\mathrm{F}$ UTURE wireless communications technologies, such as Ultra-Wideband (UWB), have several characteristics that set them apart from other wireless communications technologies. One of these characteristics is the ability to capture a few packets simultaneously [53], [56]. A Multipacket Reception (MPR) capability at the physical layer calls for the design of new Medium Access Control (MAC) protocols that are tailored for this capability [20], [53]. The combination of MPR at the physical layer and a tailored MAC layer has the potential to significantly improve the network's performance [17]. Yet, recent proposals for MAC protocols for UWB networks (e.g., [14], [15], [39]) do not specifically address the potential MPR capability. Moreover, although a number of previous works provided important insights regarding the effects of MPR, some of the important characteristics of the physical layer and the need for distributed operation have not been fully considered yet. Therefore, in this paper, we focus on studying the design considerations of distributed $M A C$ protocols for wireless networks with spatially distributed nodes and MPR capability.

- G.D. Celik is with the Department of Electrical Enoineering and Computer Science, MIT, 77 Massachusetts Avenue, Room 31-011, Cambridge, MA 02139-4301. E-mail: gcelik@mit.edu.

- G. Zussman is with the Department of Electrical Engineering, Columbia University, Mail Code 4712, 500 West 120th Street, Room 1300, New York, NY 10027. E-mail: gil@ee.columbia.edu.

- W.F. Khan is with Texas Instruments, MS 8679, 12500 TI Boulevard, Dallas, TX 75243. E-mail: w-khan@ti.com.

- E. Modiano is with the Department of Aeronautics and Astronautics, MIT, 77 Massachusetts Avenue, Room 33-412, Cambridge, MA 02139-4301.

E-mail:modiano@mit.edu.

Manuscript received 6 Jan. 2009; accepted 25 June 2009; published online 29 July 2009.

For information on obtaining reprints of this article, please send e-mail to: tmc@computer.org, and reference IEEECS Log Number TMC-2009-01-0005. Digital Object Identifier no. 10.1109/TMC.2009.135.

$1536-1233 / 10 / \$ 26.00$ @ 2010 IEEE
The design of MAC protocols for wireless networks has received tremendous attention in the last four decades [6], [49]. A basic underlying assumption in the design and evaluation of legacy MAC protocols (e.g., Aloha) was that any concurrent transmission of two or more packets results in a collision and failure of all packets. This assumption does not reflect the actual situation in many wireless communications systems in which the packet with the strongest power level can be received successfully (captured) in the presence of contending transmissions. This capture effect has been extensively studied in the past [1], [2], [23], [24], [26], [27], [30], [32], [36], [45], [50], [59] (for more details, see Section 2).

Some communications systems are capable of capturing more than a single packet. Such a capability is known as Multipacket Reception capability. Previous research regarding MPR includes the works of Ghez et al. [20], [21] who studied the stability properties of slotted aloha with MPR; Tong et al. [37], [53], [56], [57] who proposed MAC protocols that take into account the MPR capability; and Nguyen et al. [43] who considered the SINR capture model of [59] in the context of MPR systems (for more details, see Section 2). Despite the recent interest in the area of MPR, numerous research challenges still exist [17]. In particular, little has been done toward the design of distributed algorithms that work effectively with spatially distributed nodes.

Under existing backoff mechanisms (e.g., CSMA/CA, used in IEEE 802.11), once a node transmits and senses a collision, it increases its contention window (i.e., decreases the transmission probability). On the other hand, following a successful transmission, it decreases the contention window. We refer to such an operation model as the Standard Model. It is well known that the Standard Model may be unfair and can cause starvation to some of the nodes [3], [4], [18], [42]. The unfairness phenomenon is more 


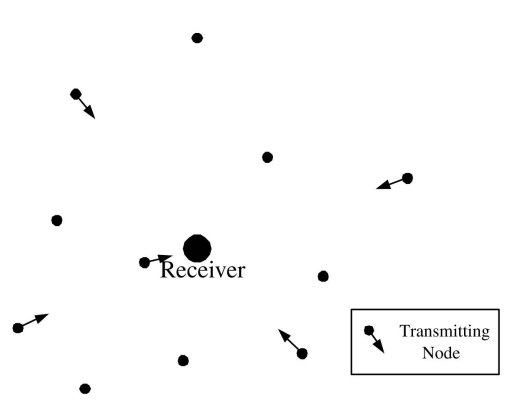

Fig. 1. Nodes distributed in a plane and a single receiver with a multipacket reception capability. Five nodes transmit simultaneously. The high-power signal from the nearby node results in failure of the four transmissions from the distant nodes. Due to the multipacket reception capability, if the nearby node does not transmit, these four transmissions can be captured.

pronounced in networks with spatially distributed nodes, since the received signals from the distant nodes are usually weaker than the signals from the nearby nodes. This may lead to starvation of distant nodes because once a nearby node captures the channel, it increases its transmission probability hence continuing to capture the channel. On the other hand, the distant nodes that failed to capture the channel will decrease their transmission probability further reducing their chances to succeed.

In networks with MPR capability, operating according to the standard backoff mechanism leads not only to unfairness, but also to significant throughput reduction. Fig. 1 illustrates an example in which one nearby node and four distant nodes attempt to transmit. ${ }^{1}$ In a typical scenario, the packet of the nearby node is captured and the others are lost due to their relatively low power levels. Accordingly, the nearby node maintains a small contention window, while the distant nodes maintain relatively large contention windows. Hence, a nearby node that captures the channel may starve several distant nodes, whose transmissions could have been captured simultaneously. In other words, instead of a potential throughput of four packets, this system has a throughput of one packet.

The above example suggests that in a network with MPR, a distributed backoff protocol should attain some fairness as an avenue to achieving higher network throughput. One possibility is to somehow increase the transmission probabilities of distant (usually failing) nodes while decreasing the transmission probabilities of nearby (usually successful) nodes. Due to the spatial distribution, the number of remote nodes is usually considerably larger than that of nearby nodes. Therefore, since a few packets can be captured simultaneously, allowing a higher transmission probability to distant nodes can increase the network throughput.

To achieve this, we define an Alternative Model in which a node decreases its transmission probability following success and increases it following failure. In order to evaluate the Standard and Alternative Models, we define a simple MAC protocol, referred to as the Generic Distributed Probabilistic (GDP) protocol. ${ }^{2}$ Under this

1. For simplicity of the example, we consider only power attenuation and ignore fading.

2. We note that our objective is to gain understanding of the effects of MPR. Therefore, this protocol does not deal with many practical challenges. protocol, each node selects one of two transmission probabilities according to its success history.

We study the performance of the GDP protocol through Markovian analysis, approximations, and simulations. Our analysis considers a single receiver (e.g., a base station) and multiple transmitters. We note that in this setting, a distributed solution is not strictly necessary. However, we focus on the design of randomized (and hence, distributed) access mechanisms that can ultimately be of use in a multihop setting where a centralized solution is less appealing. We also note that although we focus on controlling the transmission probability, other mechanisms, such as power control [29], [33], [34], [40], [50], can also be utilized in an MPR setting. However, power control mechanisms require sophisticated feedback and complex transmitters that can adjust the transmit power level dynamically on a per-packet basis. Although such mechanisms are appropriate for cellular networks, they may be more difficult to adapt to wireless (ad hoc and sensor) networks. Moreover, in a multihop setting in which a transmitter may interfere with transmissions destined to a number of receivers, the distributed power control problem is a challenging open problem even for the single-packet reception case [41].

The main contribution of this work is the thorough performance evaluation of simple generic MAC protocols in a way that enables us to gain important insights regarding the correct operating point when the network is capable of MPR. While the idea of using feedback from the channel to tune a backoff algorithm is not new, our work exploits this idea in the context of networks with spatially distributed nodes capable of MPR and shows that the tuning has to be done differently than in legacy systems capable of singlepacket reception. In particular, we provide extensive numerical results that demonstrate that the Alternative Model outperforms the Standard Model both in terms of throughput and fairness in a wide range of network scenarios. This implies that one cannot directly reuse backoff mechanisms (e.g., CSMA/CA) designed for narrow-band systems in networks with MPR capability.

This paper is organized as follows: In Section 2, we briefly review related work, and in Section 3, we describe the system model and the generic protocol. In Section 4, we provide an example that motivates the study of the Alternative Model and the GDP protocol. In Section 5, we present an analysis of the GDP protocol in a fading-free environment, and in Section 6, we extend our analysis to a fading environment. We use the GDP protocol to demonstrate the basic need to redesign the backoff mechanisms for networks with MPR capability. However, the GDP protocol is rather simplistic. Hence, in Section 7, we briefly discuss a more practical implementation of the basic idea using a simple contention window backoff mechanism similar to that used in IEEE 802.11 systems. We present an extensive numerical analysis of both the GDP protocol and the window backoff mechanism in Section 8 .

\section{Related Work}

As mentioned above, the area of MAC design has been extensively studied in the past four decades. The specific research area most relevant to our work is the one that 
focuses on the effect of capture on the design of MAC protocols. Early work in this area includes [40], [48], [50] that studied the perfect capture model in which the packet with the strongest received power is captured. A more accurate SINR-based capture model in which a single packet is captured if the SINR is above a given threshold (see Section 3) was studied in [2], [12], [28], [30]. A detailed review of the work in this field prior to 1993 can be found in Linnartz's book [32], where many variations of the SINR model have been analyzed.

Subsequent works [27], [29], [33], [59], [61] extended the results regarding the capture model and determined transmission probabilities and power control schemes for various scenarios. In particular, Zorzi and Rao [59] analyzed an Aloha system with Rayleigh fading and capture. In Section 6, we build on their results in order to analyze systems with MPR capability. As [43], [60] pointed out, in general, the extension of the results of [59] to networks with MPR capability is not immediate.

Recent work about capture focused mostly on studying its effect on IEEE 802.11 (CSMA/CA) networks. In particular, Hadzi-Velkov and Spasenovski [23], [24], [25] extended the model of Bianchi [5] to networks with capture. The effects of different distances and different power levels have been studied in [36], [45]. As mentioned above, it has been shown in [3], [4], [18], [42] that the operation of IEEE 802.11 (CSMA/CA) networks may result in unfairness which is partially due to the capture effect.

Most of the work mentioned above focused on networks in which at most a single packet can be captured at any given time. The introduction of new technologies such as UWB motivated recent research regarding MPR systems in which more than a single packet can be captured. Early work in this area includes the work of Ghez et al. [20], [21] in which the channel was modeled such that the number of successfully received packets is a random variable which depends on the number of simultaneous transmissions. In [20], [21], the effect of this MPR model on the Aloha protocol with adaptive transmission probabilities was studied. Collision resolution algorithms for networks with MPR capability that follow the model of [20] were studied in [31]. Optimal-centralized scheduling algorithms for TDMA networks with multiple channels and MPR capability were studied in [11]. Finally, the SINR capture model in which more than a single packet can be captured was studied in [26] where mostly asymptotic results were provided.

More recently, the authors of [10], [19], [35], [46] revisited the model of Ghez et al. [20]. In particular, Peh et al. [46] proposed an improved retransmission control scheme that utilizes additional feedback, MacKenzie and Wicker [35] have taken a game theoretic approach, Chan and Berger [10] analyzed an extension of the CSMA protocol, and Gau and Chen [19] proposed a multicast polling scheme based on channel state predictions. Tong et al. [37], [38], [53], [56], [57] used the model of [20] and extended the analysis in various directions. For example, Zhao and Tong [56], [57] designed new MAC protocols for networks with MPR capability that determine the set of nodes which are allowed to transmit based on the channel history and the QoS constraints.
A different approach is based on the SINR capture model. For example, Nguyen et al. [44] derived expressions for capture probability for both narrow-band (single capture) and wideband (MPR) communication systems. Luo and Ephremides [34] showed that in MPR networks, the single power level system achieves optimal throughput. Finally, the performance of slotted Aloha was analyzed in a singlehop MPR setting [16] and a multihop MPR setting [13].

In a series of recent papers, Garcia-Luna-Aceves et al. [17], [54], [55] studied the effect of various MPR models on the network capacity and energy efficiency. The results in [17], [55] are mostly asymptotic results that extend the results of [22], while Wang and Garcia-Luna-Aceves [54] provided a joint routing and scheduling approach.

Cross-layer approaches that considered the design of MAC protocols for MPR networks have been discussed in a number of recent papers. Particularly, Zheng et al. [58] proposed a physical layer MPR technique and the corresponding MAC layer which closely follows the IEEE 802.11 DCF protocol, Shekhar and Ingram [51] modeled an Aloha network with MPR capability above an OFDMA physical layer. In addition, Casari et al. [7] studied MAC design issues taking into account the implications of a multiuser detection scheme using multiple antennas, and Realp and Perez-Neira [47] proposed a joint design of physical and MAC layers that exchange relevant parameters.

Despite the recent interest in the area of MPR, the design of MAC protocols that take into account the specific characteristics of an MPR network operating according to the SINR capture model received limited attention. Moreover, little has been done toward the design of distributed algorithms that work effectively with spatially distributed nodes. In this paper, we focus on the design considerations of MAC protocols that are tailored for MPR and can remedy the throughput reduction and fairness problems arising from reusing legacy MAC protocols in networks with spatially distributed nodes.

\section{System Model and a Generic Protocol}

\subsection{Preliminaries and Model}

Consider $n$ spatially distributed nodes that communicate with a single receiver (e.g., a base station or an access point) over a slotted channel. We assume that the packets are oneslot long and the transmit power is constant (denoted by $P_{T}$ ). We assume that the propagation model includes path loss and Rayleigh fading (i.e., there exist independent and identically distributed Rayleigh fading channels between the nodes and the receiver). In such a model, the received power of a transmission from node $i$, located at distance $r_{i}$ from the receiver, is given by [26], [59]:

$$
P_{R}(i)=R^{2} K r_{i}^{-\beta} P_{T},
$$

where $R$ is a Rayleigh distributed random variable with unit power $\left(R^{2} \sim \operatorname{Exponential}(1)\right), \beta$ is the power loss exponent (typically between 2 and 6 ), and $K$ is the attenuation constant. We note that when we analyze systems without fading, we assume that $R=1$.

We use the SINR capture model [30], [32], [43], [59], defined below (also known as the Physical Model [22] and the power capture model [26]). 


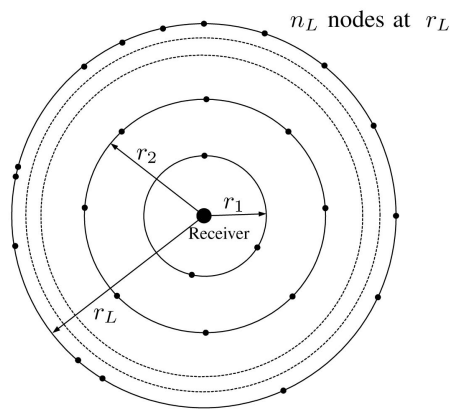

Fig. 2. Nodes deployed according to the rings model on rings whose radii are $r_{1}, r_{2}, \ldots, r_{L}$.

Definition 1 (SINR capture model). Given $k$ simultaneous transmissions, the packet of node $i$ is captured (successfully decoded) at the receiver if

$$
\operatorname{SINR}(i)=\frac{P_{R}(i)}{N+\sum_{j=1, j \neq i}^{k} P_{R}(j)}>z,
$$

where $z$ is the capture threshold ratio and $N$ is the background noise.

The background noise power level is usually much lower than the power level of the interference, and therefore, it will be neglected. For single-packet reception narrow-band systems $1 \leq z \leq 10$, whereas for wideband Multipacket Reception systems (e.g., CDMA and UWB) $z<1$ [26], [43]. Since we focus on the MPR capability, we assume that $z<1$. The maximum number of simultaneously successful transmissions is denoted by $c$. From (2), it can be seen that the maximum number of packets will be captured, if there are $c$ equal received power packets at the receiver. Hence, $c=\lceil 1 / z\rceil .^{3}$

In Section 4, we will present a simple motivating example. For that example, we will consider a simpler system that operates according to the vulnerability circle model (also known as the Protocol Model [22]), defined below.

Definition 2 (Vulnerability circle capture model [1], [48]). A transmission from distance $r$ is successful, if there are no simultaneous transmissions within a disk of radius $\alpha r(\alpha>1)$ around the receiver.

We analyze the performance of the system in various scenarios. In particular, we consider the node distribution models, defined below. ${ }^{4}$ We note that Fig. 2 illustrates an example of nodes deployed according to the rings model.

Definition 3 (Rings model). $n_{1}, n_{2}, \ldots, n_{L}$ nodes are located on $L$ rings around the receiver $\left(\sum_{i=1}^{L} n_{i}=n\right)$. The radii of these rings are denoted by $r_{1}, r_{2}, \ldots, r_{L}$, respectively.

Definition 4 (Disk model [30], [32], [59]). n nodes are randomly distributed in a disk of radius $r_{\mathrm{d}}$ and the Probability Density Function (PDF) of a node's distance from the receiver $(r)$ is denoted by $f(r)$.

3. In [26], the capture equation (2) is defined with an equality, and therefore, there: $c=1+\lfloor 1 / z\rfloor$.

4. Since nodes cannot get arbitrarily close to the receiver, when considering the SINR model, we assume that nodes are distributed such that $\min (r) \geq 1$. Specifically, in the disk model, we consider a punctured disk [27], [30].

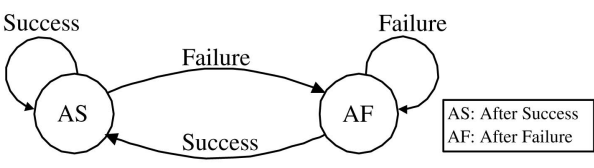

Fig. 3. The state diagram of a node under the GDP protocol.

In Section 3.2, we will present a generic protocol in which the transmission attempts are random. When analyzing this protocol, we denote the failure probability of a packet transmitted by a node located at distance $r$ from the receiver by $p_{\mathrm{f}}(r)$ and the probability of transmission by $\tau(r)$. The throughput of a node at distance $r$ is denoted by $S(r)$ and is defined as the expected number of successfully received packets per slot for that node. The overall throughput is the expected number of successfully received packets per slot throughout the system and is denoted by $S$.

Finally, in order to enable analytic performance evaluation, we make two assumptions that have been extensively used in previous works (see the reviews in [6], [49]). First, there exists a simple immediate and reliable feedback mechanism that provides the node an acknowledgement if its attempt succeeded. Second, the nodes are saturated. Namely, at each node, there is always a packet to send.

\subsection{Generic Distributed Probabilistic (GDP) Protocol}

We now define the GDP protocol. The analysis and performance evaluation of this protocol are later used to demonstrate the need to redesign MAC protocols for networks with MPR capability. Since we focus on the effects of MPR, the protocol does not deal with many practical issues and is far from providing a complete MAC solution (such as provided, for instance, by IEEE 802.11).

The operation of a node under the GDP protocol is described in the state diagram in Fig. 3. It can be seen that a node can be in two states: AS-After Success and AF-After Failure. Transitions may take place after a transmission attempt. A node moves into the AS (AF) state following a successful (failed) transmission of a packet by that node. Since transitions take place after an attempt, a node does not change its state following an idle slot.

The GDP protocol can be viewed as a two-state Aloha system that dynamically adjusts the transmission probability of each node according to its success history. When a node is in the AS (AF) state, it transmits with probability $p_{\text {ts }}$ $\left(p_{\mathrm{tf}}\right)$ at each slot, disregarding the status of the channel. The protocol is generic in the sense that it can be evaluated under any combination of $p_{\mathrm{ts}}$ and $p_{\mathrm{tf}}$. A high $p_{\mathrm{ts}}$ value corresponds to maintaining a small contention window following a successful attempt in the traditional backoff mechanisms such as the one used in IEEE 802.11. Similarly, a low $p_{\text {tf }}$ value corresponds to maintaining a large contention window following a failure. Hence, for a large value of $p_{\mathrm{ts}}$ and a small value of $p_{\mathrm{tf}}$, the protocol captures, in a very simplistic sense, the dynamic and adaptive operation of traditional backoff mechanisms. Accordingly, we refer to the operation of the GDP protocol with $p_{\mathrm{ts}}>p_{\mathrm{tf}}$ as the Standard Model and the operation with $p_{\mathrm{tf}}>p_{\mathrm{ts}}$ as the 
Alternative Model. We will show that although the Standard Model may achieve good throughput in networks with single-packet reception, and in networks with MPR capability and spatially distributed nodes, the Alternative Model usually yields higher throughput than the Standard Model. ${ }^{5}$

\section{A Motivating Example}

In this section, we analyze the performance of the GDP protocol in a simplistic scenario by an approximation method. We consider $n$ nodes distributed according to the disk model. Unlike in the rest of the paper, we assume that the system operates according to the vulnerability circle model, defined in Definition 2. According to this model, the receiver can capture a single packet. Yet, even this case provides some insight into the performance of the Standard and Alternative Models.

We first introduce an assumption that enables the derivation of approximate results. ${ }^{6}$

Definition 5 (Independence assumption). The probability that a packet transmitted by a node at distance $r$ is lost, $p_{\mathrm{f}}(r)$, is constant and is independent of the number of retransmissions suffered.

In general, when a node transmits a packet, the probability that it is lost (due to collisions) depends on other transmissions during that slot. However, similar independence assumptions have been made in the analyses of IEEE 802.11 (while taking into account the dependence on $r$ [36] or ignoring it [5]). When the number of nodes is large, the independence assumption is likely to hold. Indeed, in [8, Chapter 5], we have shown via exact analysis and simulation that results based on this assumption provide a good approximation.

From Fig. 3, it can be seen that a node changes its state from AS to AF, if it attempts to transmit and fails. Under the independence assumption, the probability of such a transition for a node at distance $r$ is $p_{\mathrm{ts}} p_{\mathrm{f}}(r)$. Similarly, a node changes its state from AF to AS, if it attempts and succeeds (i.e., with probability $\left.p_{\mathrm{tf}}\left(1-p_{\mathrm{f}}(r)\right)\right)$. Hence, the state of a specific node is described by a two-state Markov chain with the above transition probabilities. The steady-state probabilities of states AS and AF for a node at distance $r$ can be found to be:

$$
\begin{aligned}
& p_{\mathrm{AS}}(r)=\frac{p_{\mathrm{tf}}\left(1-p_{\mathrm{f}}(r)\right)}{p_{\mathrm{ts}} p_{\mathrm{f}}(r)+p_{\mathrm{tf}}\left(1-p_{\mathrm{f}}(r)\right)}, \\
& p_{\mathrm{AF}}(r)=\frac{p_{\mathrm{ts}} p_{\mathrm{f}}(r)}{p_{\mathrm{ts}} p_{\mathrm{f}}(r)+p_{\mathrm{tf}}\left(1-p_{\mathrm{f}}(r)\right)} .
\end{aligned}
$$

Accordingly, the overall transmission probability of a node at distance $r$ is given by

$$
\tau(r)=p_{\mathrm{ts}} p_{\mathrm{AS}}(r)+p_{\mathrm{tf}} p_{\mathrm{AF}}(r)=\frac{p_{\mathrm{ts}}}{1-p_{\mathrm{f}}(r)+\frac{p_{\mathrm{ts}}}{p_{\mathrm{tf}}} p_{\mathrm{f}}(r)} .
$$

A transmission from a node at distance $r$ fails, if at least one node among the $n-1$ other nodes transmits from

5. There are some cases in which the Standard Model outperforms the Alternative Model. For example, when there is a single node in the network, the Standard Model is preferable.

6. This assumption will also be used in Section 6 .

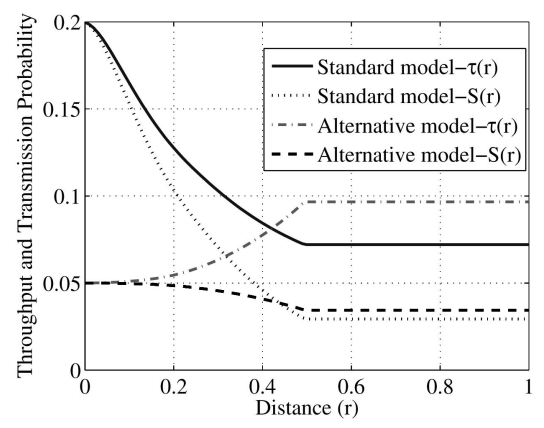

Fig. 4. The transmission probability $(\tau(r))$ and the throughput $(S(r))$ as a function of distance under the GDP protocol using the Standard Model $\left(p_{\mathrm{ts}}=0.2, p_{\mathrm{tf}}=0.05\right)$ and the Alternative Model $\left(p_{\mathrm{ts}}=0.05, p_{\mathrm{tf}}=0.2\right)$. The system operates according to the vulnerability circle model with $\alpha=2$ and Uniform distribution of 12 nodes in a disk of radius 1 .

within a disk of radius $\alpha r$ around the receiver. Under the independence assumption, the probability of this event is

$$
p_{\mathrm{f}}(r)=1-\left(1-\int_{0}^{\alpha r} \tau(x) f(x) d x\right)^{n-1} .
$$

Accordingly, the throughput of a node at distance $r$ is $S(r)=\tau(r)\left(1-p_{\mathrm{f}}(r)\right)$ and the overall throughput is $S=$ $n \int_{0}^{r_{d}} S(r) f(r) d r$.

For a given probability density function $f(r),(3)$ and (4) can be solved numerically as a system of nonlinear equations to obtain approximate values of $p_{\mathrm{f}}(r)$ and $\tau(r)$ for discrete values of $r$. Using these values, one can numerically compute $S$.

We used this method to obtain numerical results for various cases. For example, for $n=12, \alpha=2$, and $f(r)=$ $2 r \forall r \in(0,1)$ (Uniform distribution of the nodes in a disk of radius 1), Fig. 4 presents the values of $\tau(r)$ and $S(r)$ for two combinations of $p_{\mathrm{ts}}$ and $p_{\mathrm{ts}}$. One combination represents a Standard Model, while the other represents an Alternative Model.

It can be seen that under the Alternative Model, the transmission probabilities $\tau(r)$ of the distant nodes are higher than the transmission probabilities of the nearby nodes. Furthermore, the transmission probabilities of the distant nodes in the Alternative Model are higher than those of the Standard Model. This leads to a system that is more fair than the one managed by the Standard Model and that provides similar throughput values to the nodes regardless of their location. In the Alternative Model, the throughput does not decay much with the distance, whereas in the Standard Model, the throughput of distant nodes is significantly less than that of nearby nodes. Since the number of distant nodes is significantly larger than the number of nearby nodes, the overall throughput in both models is similar.

To conclude, this example demonstrates that even in a case of single-packet reception capability, the Alternative Model achieves more fair results for distant nodes and can potentially provide better throughput characteristics than the Standard Model. We naturally expect this to hold even more strongly in systems with MPR capability. Hence, in the following sections, we will provide exact and approximate analysis of the GDP protocol in various settings under 


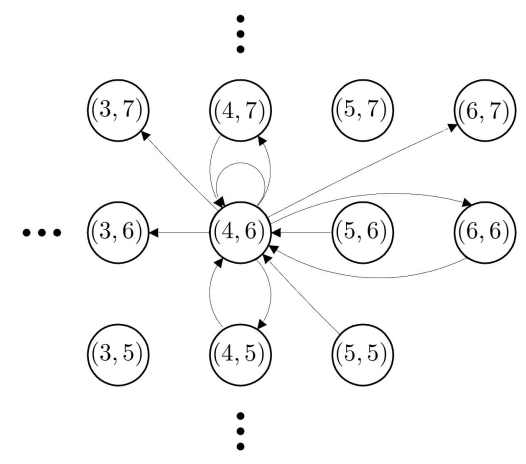

Fig. 5. Part of the Markov chain characterizing a system with two rings in which $n_{1}=6$ and $n_{2}=10$ (only some of the possible transitions to and from state $(4,6)$ are shown).

the assumption of MPR capability. We will show by numerical analysis that the Alternative Model yields more fair results and usually higher throughput compared to the Standard Model.

\section{Rings Model Without Fading}

In this section, we analyze the GDP protocol under the rings model in a system that operates according to the SINR capture model without fading. We provide exact and approximate results. We have used the exact results in order to check the accuracy of the approximation method and our simulation model (described in Section 8).

\subsection{Two Rings-Exact Analysis}

We first provide exact results for the specific case of nodes distributed on two rings. In the following sections, we will provide approximate results for more general scenarios. Consider $n_{1}$ and $n_{2}$ nodes distributed on two rings whose radii are $r_{1}$ and $r_{2}$. Using (1) and (2), it can be seen that if $r_{1}^{-\beta}>z\left((c-1) r_{1}^{-\beta}+n_{2} r_{2}^{-\beta}\right)$, the $n_{2}$ nodes on the ring at $r_{2}$ cannot generate enough interference to cause failure to a transmission from a node on the ring at $r_{1}$ (even if there are $c-1$ other transmissions from $r_{1}$ ). A simplification yields the following observation:

Observation 1. If $n_{2}<\left(r_{2} / r_{1}\right)^{\beta}\left(z^{-1}-c+1\right)$, then transmissions from the nodes on the ring at $r_{2}$ cannot cause failures to packets transmitted from the nodes on the ring at $r_{1}$.

The following can also be obtained from (1) and (2):

Observation 2. If $\left(r_{1} / r_{2}\right)^{\beta}<z$, then a transmission from a node at $r_{2}$ can be captured only when all the nodes at $r_{1}$ are silent.

For example, for $z=0.2, \beta=4, r_{1}=1$, and $r_{2}=2$, the condition in Observation 1 implies that $n_{2}<16$. In order to facilitate the exact analysis, we assume that the conditions presented in the observations above hold. Under these conditions, there is partial independence in the system.

We describe the evolution of the system under the GDP protocol by a two-dimensional Markov chain whose states are denoted by $(i, j) . i$ and $j$ denote the number of nodes on the rings at $r_{1}$ and $r_{2}$, respectively, that are in the AF state. Accordingly, the number of states in the chain is $\left(n_{1}+1\right)\left(n_{2}+1\right)$. An example of part of such a Markov chain is illustrated in Fig. 5. Each arrow represents a possible transition between states $(i, j)$ and $(l, m)$ that has some transition probability $\left(P_{(i, j) \rightarrow(l, m)}\right)$ associated with it. We group the transition probabilities according to the type of change (positive, negative, or none) in each of the two dimensions. For example, one group includes the transition probabilities for which $l>i$ and $m>j$, while another group includes the probabilities for which $l<i$ and $m=j$. Considering all the possible combinations of changes results in nine groups of transition probabilities. Below, we describe the derivation of these probabilities in three demonstrative cases.

Case I. The first case is the probability that the number of nodes on the ring at $r_{1}$ which are in the AF state decreases from $i$ to $l$ and that the number of nodes on the ring at $r_{2}$ which are in the AF state decreases from $j$ to $m$. In order for the number of nodes in the AF state to decrease from $i$ to $l$, some nodes at $r_{1}$ have to transmit. Due to the assumption that the condition presented in Observation 2 holds, the nodes on the ring at $r_{2}$ cannot succeed, if any node on the ring at $r_{1}$ transmits. Therefore, the number of nodes in the AF state cannot be simultaneously reduced on both rings. Hence, for $l<i$ and $m<j, P_{(i, j) \rightarrow(l, m)}=0$.

Case II. The second case, appearing in (5), is the probability that the number of nodes on the ring at $r_{1}$ which are in the AF state increases by $s(s \triangleq l-i>0)$ and the number of nodes on the ring at $r_{2}$ which are in the AF state increases by $k(k \triangleq m-j>0)$. Due to the assumption that the condition presented in Observation 1 holds, transmissions by the nodes on the ring at $r_{2}$ cannot cause failures to packets of the nodes on the ring at $r_{1}$. Moreover, simultaneous transmissions from the nodes on the ring at $r_{1}$ are successful as long as the number of these transmissions is at most $c$. Therefore, when $l \leq c$, the number of nodes in the AF state cannot increase to $l$, and hence, if $l \leq c$, $P_{(i, j) \rightarrow(l, m)}=0$.

On the other hand, if $l \geq c+1$, the transition probability is positive and we have to consider two subcases. The first subcase is for $s \leq c$ and is described in the second line of (5) (in Fig. 5, an example of this subcase for $c=5$ is the transition from the state $(4,6)$ to the state $(6,7))$. In such a case, there should be $s$ transmissions from the nodes on the ring at $r_{1}$ that are in the AS state (there are $n_{1}-i$ such nodes) in order for these nodes to move from the AS state to the AF state. Moreover, in order for these nodes to fail, there should be more than $c$ transmissions from nodes on the ring at $r_{1}$. This requires that there would be at least $c+1-s$ transmissions from the $i$ nodes in the AF state. Since nodes on the ring at $r_{1}$ certainly transmit in this subcase, the transmissions from the nodes on the ring at $r_{2}$ always fail. Hence, there should be $k$ transmissions from the $n_{2}-j$ nodes on the ring at $r_{2}$ that are in the AS state in order to increase the number of nodes in the AF state by $k$.

The second subcase is for $s \geq c+1$ and described in the third line of (5) (in this subcase, $l \geq c+1$ always holds, since $l=s+i$ ). Similarly to the subcase described above, there should be $s$ transmissions from the $n_{1}-i$ nodes on the ring at $r_{1}$ that are in the AS state. The transition probability is independent of the transmissions from the nodes on the ring at $r_{1}$ that are in the AF state. This is due to the fact that 
$s \geq c+1$ which implies that there are already more than $c$ transmissions from the nodes in the AS state on the ring at $r_{1}$. These transmissions will fail whether or not there is a transmission from the nodes in the AF state. Similar to the previous case, there should be $k$ transmissions from the $n_{2}-j$ nodes on the ring at $r_{2}$ that are in the AS state in order to increase the number of nodes in the AF state by $k$.

$$
\begin{aligned}
& P_{(i, j) \rightarrow(l, m)} \\
& \begin{cases}0, & \text { if } l \leq c, \\
\left(\begin{array}{c}
n_{1}-i \\
s
\end{array}\right) p_{\mathrm{ts}}^{s}\left(1-p_{\mathrm{ts}}\right)^{n_{1}-i-s} . & \\
\left(\begin{array}{c}
n_{2}-j \\
k
\end{array}\right) p_{\mathrm{ts}}^{k}\left(1-p_{\mathrm{ts}}\right)^{n_{2}-j-k} . & \\
\sum_{i}^{i}\left(\begin{array}{c}
i \\
v
\end{array}\right) p_{\mathrm{tf}}^{v}\left(1-p_{\mathrm{tf}}\right)^{i-v}, & \text { if } s \leq c \text { and } l \geq c+1, \\
\left(\begin{array}{c}
n_{1}-i \\
s
\end{array}\right) p_{\mathrm{ts}}^{s}\left(1-p_{\mathrm{ts}}\right)^{n_{1}-i-s} . & \\
\left(\begin{array}{c}
n_{2}-j \\
k
\end{array}\right) p_{\mathrm{ts}}^{k}\left(1-p_{\mathrm{ts}}\right)^{n_{2}-j-k}, & \text { if } s \geq c+1 .\end{cases}
\end{aligned}
$$

Case III. The third case, appearing in (6), is the probability that the number of nodes on the ring at $r_{1}$ which are in the AF state does not change and that the number of nodes on the ring at $r_{2}$ which are in the AF state decreases by $k(k \triangleq j-m>0)$. This happens when there are $k$ successful transmissions from the $j$ nodes on the ring at $r_{2}$ that are in the AF state. Since the maximum number of simultaneously successful transmissions is $c, P_{(i, j) \rightarrow(l, m)}=0$ for $k \geq c+1$.

$$
\begin{aligned}
& P_{(i, j) \rightarrow(l, m)} \\
& l=i, m<j \\
& = \begin{cases}0, & \text { if } k \geq c+ \\
\left(1-p_{\mathrm{ts}}\right)^{n_{1}-i}\left(1-p_{\mathrm{tf}}\right)^{i}\left(\begin{array}{l}
j \\
k
\end{array}\right) p_{\mathrm{tf}}^{k}\left(1-p_{\mathrm{tf}}\right)^{j-k} . & \\
\sum_{v=0}^{\min \left(c-k, n_{2}-j\right)}\left(\begin{array}{c}
n_{2}-j \\
v
\end{array}\right) p_{\mathrm{ts}}^{v}\left(1-p_{\mathrm{ts}}\right)^{n_{2}-j-v}, \quad \text { if } k \leq c .\end{cases}
\end{aligned}
$$

For $k \leq c$, in order for the transmissions from the nodes on the ring at $r_{2}$ to succeed, there should be no transmissions from the nodes on the ring at $r_{1}$. The term $(1-$ $\left.p_{\mathrm{ts}}\right)^{n_{1}-i}\left(1-p_{\mathrm{tf}}\right)^{i}$ in (6) represents this requirement. There should be $k$ transmissions from the $j$ nodes on the ring at $r_{2}$ that are in the $\mathrm{AF}$ state and at most $\min \left(c-k, n_{2}-j\right)$ transmissions from the $n_{2}-j$ nodes on the ring at $r_{2}$ that are in the AS state. If $n_{2}-j \leq c-k$, then the transmissions from the nodes on the ring at $r_{2}$ that are in the AS state do not affect the other nodes on $r_{2}$. However, if $n_{2}-j>c-k$, then the number of transmissions from the nodes in the AS state should be limited to $c-k$ in order not to affect the success of the nodes in the AF state. In Fig. 5, an example of this case for $c=5$ with $k \leq c$ and $n_{2}-j=c-k$ is the transition probability from the state $(4,6)$ to the state $(4,5)$.
For brevity, the equations representing the other six transition probabilities are omitted (they can be found in [8, Chapter 5]). Note that for $n_{1} \leq c$, the state of the system can be represented by a one-dimensional Markov chain whose states denote the number of nodes on the ring at $r_{2}$ that are in the AF state (the transition probabilities of this one-dimensional Markov chain can be found in [8, Appendix B]). It can be shown that by letting $i=0$ and $l=0$, the two-dimensional Markov chain, described above, reduces to this one-dimensional Markov chain. Furthermore, for the simpler case in which all the nodes are at the same distance from the receiver (e.g., for $n_{1}=0$ ), the state of the system can be represented by a one-dimensional Markov chain whose states denote the number of nodes in the AF state. Such a system was analyzed in [49, Chapter 3.3], where nodes after failed transmission attempts were termed backlogged nodes. When all the nodes are on a single ring, our two-dimensional Markov chain reduces to the Markov chain of [49].

Given the transition probabilities, the steady-state probabilities (denoted by $p(i, j)$ ) can be obtained by solving a set of linear equations. Once the steady-state probabilities are obtained, the throughput of the system can be calculated as follows: We denote by $S_{\mathrm{rg}}(m, i)$ the overall throughput of the nodes on a ring that has $m$ nodes, of which $i$ are in the AF state. Assuming that there is a single ring:

$$
\begin{aligned}
& S_{\mathrm{rg}}(m, i)=\sum_{f=0}^{\min (c, i)} \sum_{s=0}^{\min (c-f, m-i)}(f+s) . \\
& \left(\begin{array}{c}
i \\
f
\end{array}\right) p_{\mathrm{tf}}^{f}\left(1-p_{\mathrm{tf}}\right)^{i-f}\left(\begin{array}{c}
m-i \\
s
\end{array}\right) p_{\mathrm{ts}}^{s}\left(1-p_{\mathrm{ts}}\right)^{m-i-s} .
\end{aligned}
$$

The two summations are for the nodes at the two states ( $f$ is the index for the nodes at AF and $s$ is for the nodes at AS). Since at most $c$ packets can be captured simultaneously, the summations are bounded not only by the number of nodes in the relevant state, but also by $c$ and $c-f$.

Due to our assumptions, the success of nodes on the ring at $r_{1}$ is not affected by transmissions of nodes at $r_{2}$, and therefore, the throughput of these nodes can be computed as if there is a single ring. Moreover, nodes on the ring at $r_{2}$ succeed only if nodes on $r_{1}$ are silent. Therefore, in such a case, the nodes on $r_{2}$ can be treated as nodes on a single ring. Accordingly, the overall throughput is given by

$S=\sum_{i=0}^{n_{1}} \sum_{j=0}^{n_{2}} p(i, j)\left(S_{\mathrm{rg}}\left(n_{1}, i\right)+\left(1-p_{\mathrm{ts}}\right)^{n_{1}-i}\left(1-p_{\mathrm{tf}}\right)^{i} S_{\mathrm{rg}}\left(n_{2}, j\right)\right)$,

where $\left(1-p_{\mathrm{ts}}\right)^{n_{1}-i}\left(1-p_{\mathrm{tf}}\right)^{i}$ is the probability that the nodes on $r_{1}$ are silent.

\subsection{Multiple Rings-Approximate Analysis}

The exact analysis is quite cumbersome even for two rings. Hence, we present an approximation method that allows us to obtain results for a large number of rings. When the conditions in observations 1 and 2 are satisfied, the state of the nodes at $r_{1}$ is independent of the actions of nodes at $r_{2}$. Nodes at $r_{1}$ are, of course, not independent of each other. The dependence of the nodes at $r_{2}$ on the nodes at $r_{1}$ is limited to the event that "no transmission occurs from nodes at $r_{1}$. " Assuming that such a partial independence 
between the rings exists even in a system with multiple rings, we now develop a method in which we use different Markov chains to characterize the states of the nodes on the different rings. The state of each Markov chain is the number of nodes in the AF state on that ring. Within the rings, these Markov chains capture the dependence between the nodes. The interaction between the rings is captured by the following independence assumption:

Definition 6 (Interring independence assumption). For a node on a ring at $r_{j}$, the probability that a node on the ring at $r_{i}(i \neq j)$ transmits $\left(\tau\left(r_{i}\right)\right)$ is constant and is independent of the states of the nodes on the rings at $r_{h}(h \neq i)$.

In general, the probability that a node transmits depends on its success history and the operations of the other nodes. However, as mentioned above, similar independence assumptions have been made in the analyses of IEEE 802.11 [5], [36]. We note that since we assume that there is dependence between the nodes on each ring, the assumption is weaker than the assumptions made in the past and in Definition 5.

We wish to limit the possible interactions between the different rings, and thereby, between the different Markov chains. Namely, we want that for a ring at $r_{i}$, the effective $\tau\left(r_{j}\right) \forall r_{j}>r_{i}$ will be zero. For example, if the condition in Observation 1 holds, the nodes at $r_{1}$ are not affected by transmissions at $r_{2}$. Therefore, in the derivation of the Markov chain of the ring at $r_{1}$, we can assume that $\tau\left(r_{2}\right)=0$. In order to limit the interactions, the conditions at observations 1 and 2 have to hold in addition to a number of other conditions. In the following observations, we demonstrate the conditions for the case of three rings. We then outline the results for this case.

The following observation is derived from (1) and (2) in a very similar way to the derivation of Observation 1:

Observation 3. If $n_{3}<\left(r_{3} / r_{2}\right)^{\beta}\left(z^{-1}-c+1\right)$, then transmissions from the nodes on the ring at $r_{3}$ cannot cause failures to packets transmitted from the nodes on the ring at $r_{2}$.

Using (1) and (2), it can be seen that if $r_{1}^{-\beta}>$ $z\left((c-1) r_{1}^{-\beta}+n_{2} r_{2}^{-\beta}+n_{3} r_{3}^{-\beta}\right)$ holds, the $n_{2}$ and $n_{3}$ nodes on the rings at $r_{2}$ and $r_{3}$ cannot generate enough interference to cause failure to a transmission from a node on the ring at $r_{1}$ (even if there are $c-1$ other transmissions from $r_{1}$ ). A simplification yields the following observation:

Observation 4. If $n_{2}<\left(r_{2} / r_{1}\right)^{\beta}\left(z^{-1}-c+1\right)-n_{3}\left(r_{2} / r_{3}\right)^{\beta}$, then transmissions from the nodes on the rings at $r_{2}$ and $r_{3}$ cannot cause failures to packets transmitted from the nodes on the ring at $r_{1}$.

The following observation can be obtained in a similar way to Observation 2:

Observation 5. If $\left(r_{2} / r_{3}\right)^{\beta}<z$, then a transmission from a node at $r_{3}$ can be captured only when all the nodes at $r_{2}$ and at $r_{1}$ are silent.

For example, for $z=0.2$ and $\beta=4$, the values $r_{1}=1$, $r_{2}=2, r_{3}=4, n_{2} \leq 15$, and $n_{3} \leq 15$ satisfy the conditions in Observations 1-5. Assuming that the conditions in Observations 1-5 are satisfied, we obtain the transition probabilities of the Markov chains as follows: For a Markov chain of a given ring, there are three possible transitions in every time slot: the number of nodes in the AF state can increase, decrease, or stay the same. We associate a transition probability with each of these events. For brevity, we provide two demonstrative cases (the complete description can be found in [8]).

The first case is a transition probability for the Markov chain of the nodes at $r_{1}$. Recall that due to our assumptions, nodes on the ring at $r_{1}$ are not affected by nodes on other rings. Specifically, we denote by $P_{i \rightarrow(i-s)}$, the probability that the number of nodes in the AF state decreases from $i$ to $i-s$. In this case, there are $0<s \leq i$ successful transmissions from the $i$ nodes that are in the AF state. Since the maximum number of simultaneously successful transmissions is $c$, for $s \geq c+1, P_{i \rightarrow(i-s)}=0$. If $s \leq c$, then there should be $s$ transmissions from the $i$ nodes in the AF state and up to $c-s$ transmissions from the $n_{1}-i$ nodes in the AS state. Accordingly, for $s \leq c$ :

$$
\begin{aligned}
P_{i \rightarrow(i-s)}= & \left(\begin{array}{c}
i \\
s
\end{array}\right) p_{\mathrm{tf}}^{s}\left(1-p_{\mathrm{tf}}\right)^{i-s} . \\
& \sum_{v=0}^{\min \left(c-s, n_{1}-i\right)}\left(\begin{array}{c}
n_{1}-i \\
v
\end{array}\right) p_{\mathrm{ts}}^{v}\left(1-p_{\mathrm{ts}}\right)^{n_{1}-i-v} .
\end{aligned}
$$

The second case, appearing in (9), is a transition probability for the Markov chain of the ring at $r_{2}$. It is the probability that the number of nodes in the AF state increases from $j$ to $m$. This can happen due to $k$ ( $k \triangleq m-j$ ) failed transmissions from the $n_{2}-j$ nodes that are in the AS state. If $m \leq c$, nodes at $r_{2}$ fail only if there is at least one transmission from the ring at $r_{1}$. Under the Interring Independence Assumption, this happens with probability $\left(1-\left(1-\tau\left(r_{1}\right)\right)^{n_{1}}\right)$, where $\tau\left(r_{1}\right)$ is the expected transmission probability of a node on the ring at $r_{1}$ computed using the Markov chain of that ring. When $m \geq c+1$, in addition to the failures due to the transmissions from the ring at $r_{1}$, nodes on the ring at $r_{2}$ can fail, if there are more than $c$ transmissions from that ring. This second contribution occurs when there is no transmission from the ring at $r_{1}, k$ transmissions from the $n_{1}-i$ nodes on the ring at $r_{2}$ that are in the AS state, and at least $c+1-k$ transmissions from the $i$ nodes at $r_{2}$ that are in the AF state. If $k \geq c+1$, all the transmissions from the ring at $r_{2}$ will fail. In this case, the transition probability is the probability that $k$ nodes in the AS state will transmit.

Due to the decoupling between the Markov chains, the approximation method is significantly easier to formulate than the exact method presented in Section 5.1. In particular, the derivations of the transition probabilities in the Markov chains of the different rings are very similar to each other. Given the transition probabilities, the steadystate probabilities of each of these Markov chains $\left(p_{1}(i)\right.$, $p_{2}(j)$, and $\left.p_{3}(h)\right)$ can be obtained by solving a set of linear equations. Once they are obtained, the throughput of the system can be obtained in a similar way to the derivation of (8) and by using $S_{\mathrm{rg}}(m, i)$ (defined in (7)). It is given by the following expression which is explained in detail below: 


$$
\begin{aligned}
S= & \sum_{i=0}^{n_{1}} \sum_{j=0}^{n_{2}} \sum_{h=0}^{n_{3}} p_{1}(i) p_{2}(j) p_{3}(h)\left(S_{\mathrm{rg}}\left(n_{1}, i\right)\right. \\
& +\left(1-p_{\mathrm{ts}}\right)^{n_{1}-i}\left(1-p_{\mathrm{tf}}\right)^{i} S_{\mathrm{rg}}\left(n_{2}, j\right) \\
& \left.+\left(1-p_{\mathrm{ts}}\right)^{n_{1}+n_{2}-i-j}\left(1-p_{\mathrm{tf}}\right)^{i+j} S_{\mathrm{rg}}\left(n_{3}, h\right)\right) .
\end{aligned}
$$

Due to the Interring Independence Assumption, the probability of having $i, j$, and $h$ nodes in the AF states on the rings at $r_{1}, r_{2}$, and $r_{3}$, respectively, is $p_{1}(i) p_{2}(j) p_{3}(h)$. Due to our assumptions, the nodes on the ring at $r_{1}$ are not affected by the nodes on other rings, and therefore, their throughput can be computed using $S_{\mathrm{rg}}(m, i)$ as if there is a single ring. Nodes on the ring at $r_{2}$ succeed, only if the nodes at $r_{1}$ are silent (the probability of this event is $\left.\left(1-p_{\mathrm{ts}}\right)^{n_{1}-i}\left(1-p_{\mathrm{tf}}\right)^{i}\right)$. Similarly, the nodes at $r_{3}$ succeed, only if the nodes on the rings at $r_{1}$ and $r_{2}$ are silent (i.e., with probability $\left.\left(1-p_{\mathrm{ts}}\right)^{n_{1}+n_{2}-i-j}\left(1-p_{\mathrm{tf}}\right)^{i+j}\right)$.

We verified the accuracy of the approximation by comparing its results for the two rings case with the exact results. While the results for the $n_{1}=1, n_{2}=5$ and $n_{1}=2$, $n_{2}=10$ cases were the same up to three decimal places, the results for the $n_{1}=6, n_{2}=10$ case were within 1.6 percent of the exact results. We also compared the approximate results for the three rings case to results obtained by the simulation model described in Section 8. In all the considered cases, the approximate results were within 2.2 percent of the simulation results.

$$
\begin{aligned}
& P_{j \rightarrow m}\left(\begin{array}{c}
\left(1-\left(1-\tau\left(r_{1}\right)\right)^{n_{1}}\right) \\
\left(\begin{array}{c}
n_{2}-j \\
k
\end{array}\right) p_{\mathrm{ts}}^{k}\left(1-p_{\mathrm{ts}}\right)^{n_{2}-j-k}, \quad \text { if } m \leq c, \\
\left(1-\left(1-\tau\left(r_{1}\right)\right)^{n_{1}}\right)\left(\begin{array}{c}
n_{2}-j \\
k
\end{array}\right) . \\
p_{\mathrm{ts}}^{k}\left(1-p_{\mathrm{ts}}\right)^{n_{2}-j-k}+ \\
\left(1-\tau\left(r_{1}\right)\right)^{n_{1}}\left(\begin{array}{c}
n_{2}-j \\
k
\end{array}\right) . \\
p_{\mathrm{ts}}^{k}\left(1-p_{\mathrm{ts}}\right)^{n_{2}-j-k} \cdot \\
\sum_{j=c+1-k}^{j}\left(\begin{array}{l}
j \\
v
\end{array}\right) p_{\mathrm{tf}}^{v}\left(1-p_{\mathrm{tf}}\right)^{j-v}, \quad \text { if } k \leq c \text { and } m \geq c+1, \\
\left(\begin{array}{c}
n_{2}-j \\
k
\end{array}\right) p_{\mathrm{ts}}^{k}\left(1-p_{\mathrm{ts}}\right)^{n_{2}-j-k}, \quad \text { if } k \geq c+1 .
\end{array}\right.
\end{aligned}
$$

\section{Rings Model With Fading}

We continue to study the performance of the GDP protocol under the rings model. Unlike in the previous section, we now assume that the propagation model includes Rayleigh fading. This assumption allows us to obtain approximate results for a large number of rings without using Markov chains. We also use the independence assumption which was introduced in Definition 5. Under this assumption, the transmission probability of a node at distance $r$ was derived in (3). Recall that in the rings model, there are $n_{1}, n_{2}, \ldots, n_{L}$ nodes on rings of radii $r_{1}, r_{2}, \ldots, r_{L}$, respectively. In such a case, (3) can be rewritten as follows to provide the transmission probability of a node on the ring at $r_{i}$ :

$$
\tau\left(r_{i}\right)=\frac{p_{\mathrm{ts}}}{1-p_{\mathrm{f}}\left(r_{i}\right)+\frac{p_{\mathrm{ts}}}{p_{\mathrm{tf}}} p_{\mathrm{f}}\left(r_{i}\right)} \quad \forall i \quad 1 \leq i \leq\{1,2, \ldots, L\} .
$$

We define $p_{c, j}\left(r_{i} \mid r_{1}, r_{2}, \ldots, r_{L} ; k_{1}, k_{2}, \ldots, k_{L}\right)$ as the probability that a packet from a node at distance $r_{i}$ is captured, given that there are $j$ interferers distributed such that there are $k_{1}, k_{2}, \ldots, k_{i}, \ldots, k_{L}$ interferers at distances $r_{1}, r_{2}, \ldots$, $r_{i}, \ldots, r_{L}$, respectively $\left(j=\sum_{m=1}^{L} k_{m}\right) .^{7}$ Using the approximation assumption and considering all possible interferer configurations, we derive in (11) the failure probability of a transmission from distance $r_{i}$ for all the values of $i .^{8}$ In order to be able to numerically solve the set of $2 L$ nonlinear equations, given by (10) and (11), we now need to obtain $p_{c, j}\left(r_{i} \mid r_{1}, r_{2}, \ldots, r_{L} ; k_{1}, k_{2}, \ldots, k_{L}\right)$.

$p_{\mathrm{f}}\left(r_{i}\right)=$

$\sum_{k_{1}=0}^{n_{1}} \sum_{k_{2}=0}^{n_{2}} \ldots \sum_{k_{i}=0}^{n_{i}-1} \ldots \sum_{k_{L}=0}^{n_{L}}\left(\begin{array}{l}n_{1} \\ k_{1}\end{array}\right) \tau\left(r_{1}\right)^{k_{1}}\left(1-\tau\left(r_{1}\right)\right)^{n_{1}-k_{1}}$.

$\left(\begin{array}{l}n_{2} \\ k_{2}\end{array}\right) \tau\left(r_{2}\right)^{k_{2}}\left(1-\tau\left(r_{2}\right)\right)^{n_{2}-k_{2}} \ldots$

$\left(\begin{array}{c}n_{i}-1 \\ k_{i}\end{array}\right) \tau\left(r_{i}\right)^{k_{i}}\left(1-\tau\left(r_{i}\right)\right)^{n_{i}-k_{i}-1} \ldots$

$\left(\begin{array}{c}n_{L} \\ k_{L}\end{array}\right) \tau\left(r_{L}\right)^{k_{L}}\left(1-\tau\left(r_{L}\right)\right)^{n_{L}-k_{L}}$.

$\left(1-p_{c, j}\left(r_{i} \mid r_{1}, r_{2}, \ldots, r_{L} ; k_{1}, k_{2}, \ldots, k_{L}\right)\right) \forall i \in\{1,2, \ldots, L\}$.

In order to derive $p_{c, j}\left(r_{i} \mid r_{1}, r_{2}, \ldots, r_{L} ; k_{1}, k_{2}, \ldots, k_{L}\right)$, we apply a result of Zorzi and Rao [59] who analyzed an Aloha system with Rayleigh fading and capture. We define $p_{c, j}\left(r_{0} \mid r_{1}, r_{2}, \ldots, r_{j}\right)$ as the probability that the packet from a node at distance $r_{0}$ is captured in the presence of $j$ other transmissions from distances $r_{1}, r_{2}, \ldots, r_{j}$. It is given in [59] as

$$
p_{c, j}\left(r_{0} \mid r_{1}, r_{2}, \ldots, r_{j}\right)=\prod_{m=1}^{j} \frac{1}{1+z\left(\frac{r_{0}}{r_{m}}\right)^{\beta}} .
$$

Using (12) and combining the terms with the same distance, we obtain

$$
p_{c, j}\left(r_{i} \mid r_{1}, r_{2}, \ldots, r_{L} ; k_{1}, k_{2}, \ldots, k_{L}\right)=\prod_{m=1}^{L}\left[\frac{1}{1+z\left(\frac{r_{i}}{r_{m}}\right)^{\beta}}\right]^{k_{m}} .
$$

Equation (13) can now be used within (11). Then, (10) along with (11) can be solved numerically. Using the numerical solutions, one can obtain the overall throughput $S=\sum_{i=1}^{L} n_{i} \tau\left(r_{i}\right)\left(1-p_{\mathrm{f}}\left(r_{i}\right)\right)$. We used Matlab to solve the equations and obtain numerical results. We verified their accuracy using the simulation model described in Section 8. While in almost all the cases, the approximate results were within 2.6 percent of the simulation results, and a few cases were within 4.6 percent.

7. Note that $0 \leq k_{i} \leq n_{i}-1$ and $0 \leq k_{m} \leq n_{m} \forall m \neq i$.

8. Note that this is a generalization of the derivation in [45] of the failure probability in a system with two power levels. 


\section{Generic Distributed Contention Window (GDCW) PROTOCOL}

We now present the GDCW protocol, which is potentially easier to implement compared to the GDP protocol and can be operated according to the Standard and Alternative Models. Unlike the GDP protocol, the GDCW protocol does not easily lend itself to analysis. Hence, we evaluate its performance via simulation.

Similarly to the GDP protocol, nodes operate according to the state machine in Fig. 3. Namely, nodes are assigned contention windows of different sizes according to their success or failure in the previous attempt. When a node enters an AS state, it is assigned a contention window of size $w_{\mathrm{s}}$ slots. It then selects a backoff interval $B O$ uniformly in $\left[0, w_{\mathrm{s}}\right]$ and retransmits after waiting for $B O$ slots, disregarding the status of the channel. Similarly, when a node enters the AF state, it selects a backoff interval uniformly in $\left[0, w_{\mathrm{f}}\right]$ and retransmits at the end of this interval.

The GDCW protocol is generic in the sense that it can be evaluated under any combination of $w_{\mathrm{f}}$ and $w_{\mathrm{s}}$. For a large value of $w_{\mathrm{f}}$ and a small value of $w_{\mathrm{s}}$, the protocol captures, in a very simplistic sense, the operation of backoff mechanisms such as one used in IEEE 802.11. Hence, we refer to the operation with $w_{\mathrm{f}}>w_{\mathrm{s}}$ as the Standard Model. When $w_{\mathrm{s}}>w_{\mathrm{f}}$, we refer to the scheme as the Alternative Model.

\section{Performance Evaluation}

We used the analytic methods, described in Sections 5 and 6 along with extensive simulation experiments to evaluate the performance of the GDP and GDCW protocols under the Standard Model and the Alternative Model. Analytic results can be obtained for the GDP protocol under the rings model, and therefore, we present analytic results for that case. We also present simulation results for both protocols for the more realistic disk model. Extensive additional numerical results can be found in [8]. All the reported results are obtained under the SINR model with the assumption that $N=0$ and $\beta=4$. Throughout Sections 8.2-8.5, we assume that $z=0.2$ (i.e., $c=5$ - at most five packets can be captured simultaneously). In Section 8.6, we study the sensitivity of the results to changes in $z$. As mentioned in Section 3, when considering the disk model, we assume that nodes are distributed in a punctured disk and that the nodes' distribution is Uniform. Namely, $f(r)=2 r /\left(r_{d}^{2}-r_{0}^{2}\right), r_{0} \leq r \leq r_{d}$. In particular, in the reported experiments, $r_{0}=1$ and $r_{d}=10$.

\subsection{Simulation Model}

The simulation model was developed in C. It allows operating the system in different scenarios (e.g., rings model, disk model, with and without fading, etc.) and according to the different protocols. We verified the correctness of the simulation model in a number of ways. For example, we compared results obtained via simulation for the GDP protocol with the rings model to exact results obtained by the method described in Section 5.1. In all the cases, the simulation results were within 1.5 percent of the exact results. We also compared simulation results obtained for the GDP protocol with the disk model and fading to

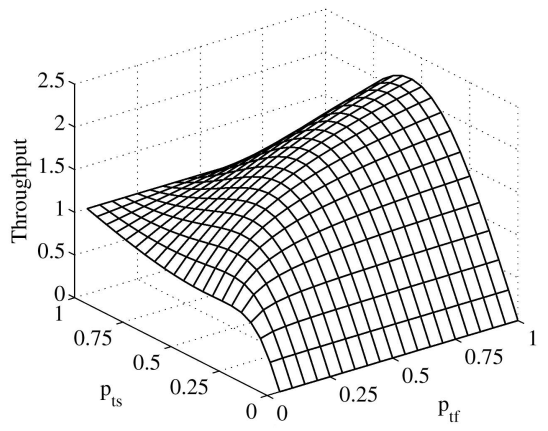

(a)

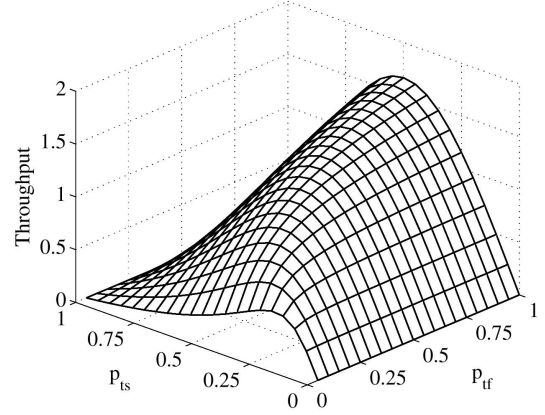

(b)

Fig. 6. (a) The overall throughput $(S)$ and (b) the throughput of the distant nodes. The system operates under the GDP protocols in a fading-free environment and the nodes are distributed according to the rings model with $r_{1}=1, r_{2}=2, n_{1}=1$, and $n_{2}=5$.

numerical results presented in [27, Figs. 4, 5, and 6] for the special case of $p_{\mathrm{tf}}=p_{\mathrm{ts}}$. Our simulation results were within 2 percent of the results in [27]. As mentioned in Sections 5.2 and 6 , once the performance of the simulation model had been verified, we also used it in order to check the accuracy of the approximation methods.

In the reported simulation results, for each data point, the simulation length was 100,000 slots. Since in the disk model, nodes are randomly placed, results presented for that model are averaged over 40 different experiments. In each of these experiments, nodes are placed differently.

\subsection{Rings Model without Fading}

We now present exact and approximate results regarding the performance of the GDP protocol and simulation results regarding the performance of the GDCW protocol.

Fig. 6a presents the overall throughput under the GDP protocol with nodes on two rings. The maximum throughput of 2.195 is obtained for $p_{\mathrm{ts}}=0.55$ and $p_{\mathrm{tf}}=1$. At that point, $p_{\mathrm{tf}}$ is larger than $p_{\mathrm{ts}}$, implying that the Alternative Model outperforms the Standard Model. Fig. 6b shows the total throughput obtained in this case by the nodes at distance $r_{2}=2$. While the distant nodes achieve very low throughput in the Standard Model, they manage to achieve high throughput in the Alternative Model. At the operating point in which the overall system throughput is maximized, the throughput obtained by a distant node is 0.33 , while the throughput obtained by a nearby node is 0.55 . Hence, the Alternative Model not only improves the performance in terms of overall throughput, but also provides some degree of fairness. 


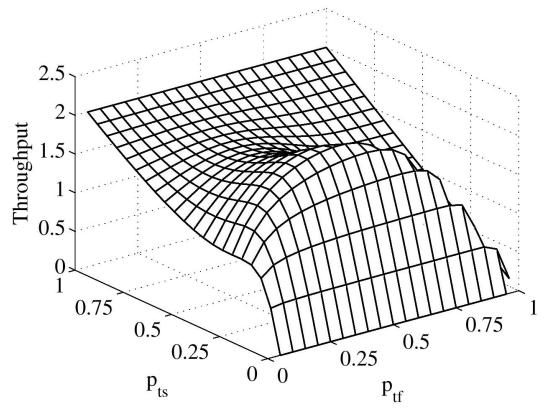

Fig. 7. The overall throughput $(S)$ under the GDP protocol in a fadingfree environment. The nodes are distributed according to the rings model with $r_{1}=1, r_{2}=2, n_{1}=2$, and $n_{2}=10$.

Fig. 7 presents the throughput for the case in which $n_{1}=$ 2 and $n_{2}=10$. The maximum throughput of $S=2.051$ is obtained for $p_{\mathrm{ts}}=0.25$ and $p_{\mathrm{tf}}=0.50$ (i.e., by the Alternative Model). We note that according to the numerical results, the throughput of the distant nodes (on $r_{2}$ ) is high under the Alternative Model, while it tends to 0 when $p_{\text {ts }}$ tends to 1 . Hence, although the Standard Model operating point of $p_{\mathrm{ts}}=1$ has relatively high overall throughput, it is unfair.

The overall throughput under the GDP protocol with nodes on three rings is presented in Fig. 8a. The maximum throughput is obtained for $p_{\mathrm{ts}}=0.35$ and $p_{\mathrm{tf}}=1$. This again demonstrates that the Alternative Model outperforms the Standard Model. Furthermore, at this maximum point, the nodes at different distances are not starved $(S(1)=0.350, S(2)=0.295, S(3)=0.154)$. Fig. $8 \mathrm{~b}$ shows the

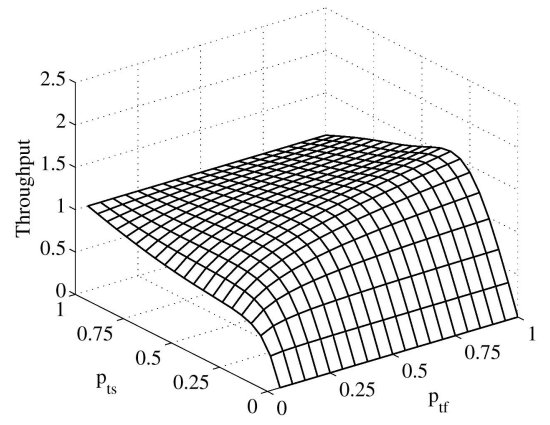

(a)

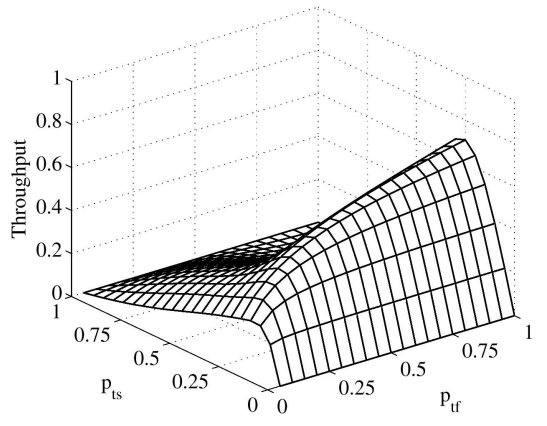

(b)

Fig. 8. (a) The overall throughput $(S)$ and (b) the throughput of the nodes at $r_{3}$, under the GDP protocol in a fading-free environment. The nodes are distributed according to the rings model with $r_{1}=1, r_{2}=2, r_{3}=4$, $n_{1}=1, n_{2}=2$, and $n_{3}=4$.

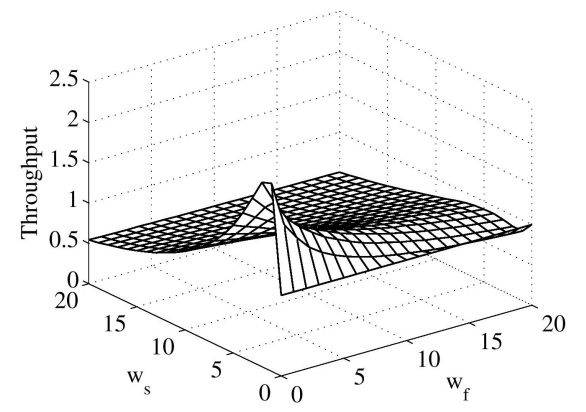

Fig. 9. The throughput $(S)$ under the GDCW protocol in a fading-free environment. The nodes are distributed according to the rings model with $r_{1}=1, r_{2}=2, n_{1}=1$, and $n_{2}=5$.

total throughput of the $n_{3}$ nodes at $r_{3}$. It can be seen that these distant nodes benefit from low values of $p_{\mathrm{ts}}$, which reduce the chances of nearby nodes to capture the channel, and high values of $p_{\mathrm{tf}}$, which increase the number of their retransmission attempts.

Up to now, we reported analytic results regarding the GDP protocol. We also evaluated the performance of the GDCW protocol via simulation. Such results for the case in which $r_{1}=1, r_{2}=2, n_{1}=1$, and $n_{2}=5$ are shown in Fig. 9 . The figure demonstrates that the throughput is maximized when $w_{\mathrm{f}}<w_{\mathrm{s}}$. Namely, the Alternative Model is beneficial for the GDCW protocol as well. A similar observation can be made by viewing the graph in Fig. 10, which has two local optimums. We note that in both of these optimums, the distant nodes are not starved.

\subsection{Rings Model with Fading}

We used the approximation method described in Section 6 to obtain numerical results regarding the performance of the GDP protocol in a system with fading in which the nodes are distributed according to the rings model. For example, Fig. 11 shows the overall throughput for a system with three rings. We observe that, as before, the throughput is maximized by large values of $p_{\mathrm{tf}}$ and small values of $p_{\mathrm{ts}}$. Once fading is considered, the received power of a transmission from a nearby node is not necessarily stronger than the power of a transmission from a distant node. However, this does not significantly change the fact that the Alternative Model still outperforms the Standard Model.

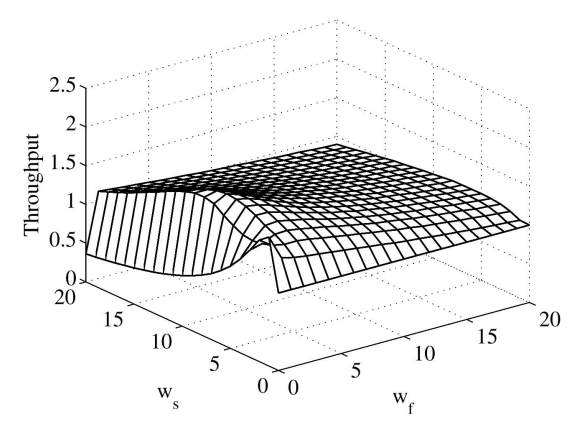

Fig. 10. The throughput $(S)$ under the GDCW protocol in a fading-free environment. The nodes are distributed according to the rings model with $r_{1}=1, r_{2}=2, r_{3}=3, n_{1}=1, n_{2}=3$, and $n_{3}=9$. 


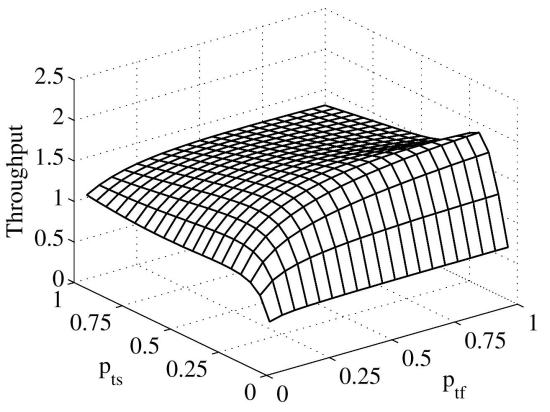

Fig. 11. The throughput $(S)$ under the GDP protocol in a system with fading. The nodes are distributed according to the rings model with $r_{1}=1, r_{2}=2, r_{3}=3, n_{1}=1, n_{2}=3$, and $n_{3}=9$.

\subsection{Disk Model without Fading}

We used the simulation model to obtain numerical results for the case in which nodes are distributed according to the disk model and there is no fading. Figs. 12a and $12 \mathrm{~b}$ show the throughput under the GDP and GDCW protocols. In both figures, there are two local optimums. One of them represents the Alternative model and the other the Standard Model. However, as before, under the Standard model, distant nodes are usually starved. Therefore, the Alternative Model is preferable. It can be seen that the results obtained for the disk model are similar to the results obtained for the rings model. Hence, the rings model provides a good approximation, while lending itself to analytic performance evaluation.

\subsection{Disk Model with Fading}

In Fig. 13, we present simulation results for a system with fading in which nodes are distributed according to the disk

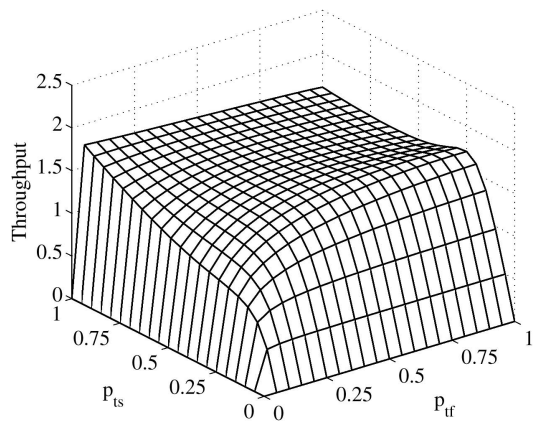

(a)

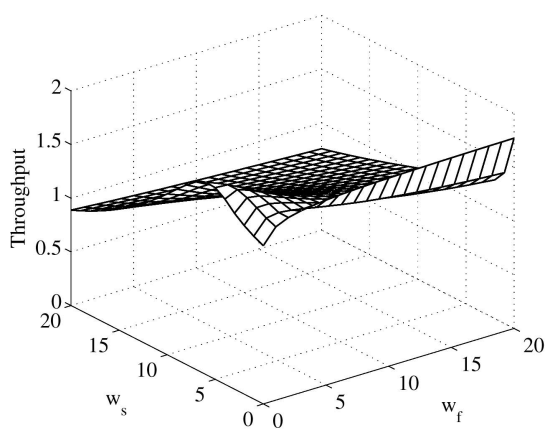

(b)

Fig. 12. The throughput $(S)$ under (a) the GDP protocol and (b) the GDCW protocol in a fading-free environment. Ten nodes are distributed according to the disk model.

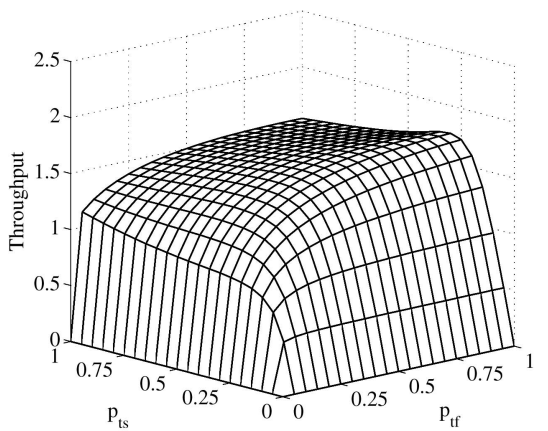

(a)

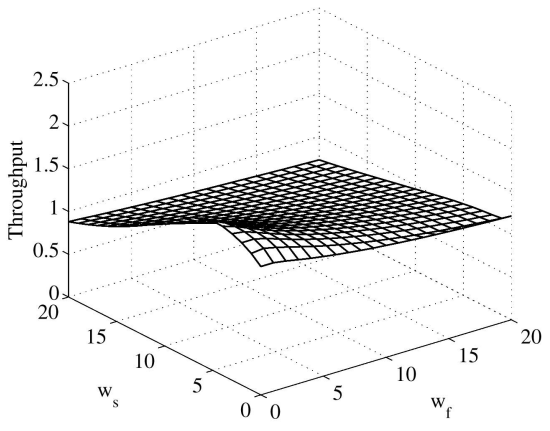

(b)

Fig. 13. The throughput $(S)$ under (a) the GDP protocol and (b) the GDCW protocol in a system with fading. Ten nodes are distributed according to the disk model.

model. It can be seen that the results are similar to the results for the nonfading case. Since the fading effect somehow averages the transmission powers, the nearby nodes do not always succeed and distant nodes may succeed despite transmissions of nearby nodes. Hence, the local optimums are not as strict as in the fading-free case. Moreover, unlike in the fading-free case, there is no local optimum corresponding to the Standard Model.

\subsection{Sensitivity to the Capture Threshold ( $z$ )}

We now present the throughput variation as a result of changes in the capture threshold $z$. We consider the GDP protocol in a system with fading in which 10 nodes are distributed according to the disk model. The values of the overall throughput for the cases of $z=0.1,0.3,0.5$, and 0.7 are presented in Fig. 14. We observe that the cases in which $z=0.1$ and $z=0.3$ yield similar throughput figures to the case in which $z=0.2$ (Fig. 13a). Hence, the results are not very sensitive to changes in $z$. As $z$ gets closer to 1 , the throughput graph tends to have two local maximums. This is due to the fact that as $z$ increases, the receiver is more likely to capture only a single packet. In such a case, giving higher priority to the node with the strongest power level results in a relatively high throughput. This corresponds to having a high $p_{\text {ts }}$ value. Nevertheless, we see that low $p_{\text {ts }}$ values also result in similar throughput levels. Namely, the Alternative Model has comparable throughput to the Standard Model even when the receiver tends to lose the MPR capability.

\section{Conclusions}

The Multipacket Reception capability has the potential to significantly improve the performance of wireless networks. 

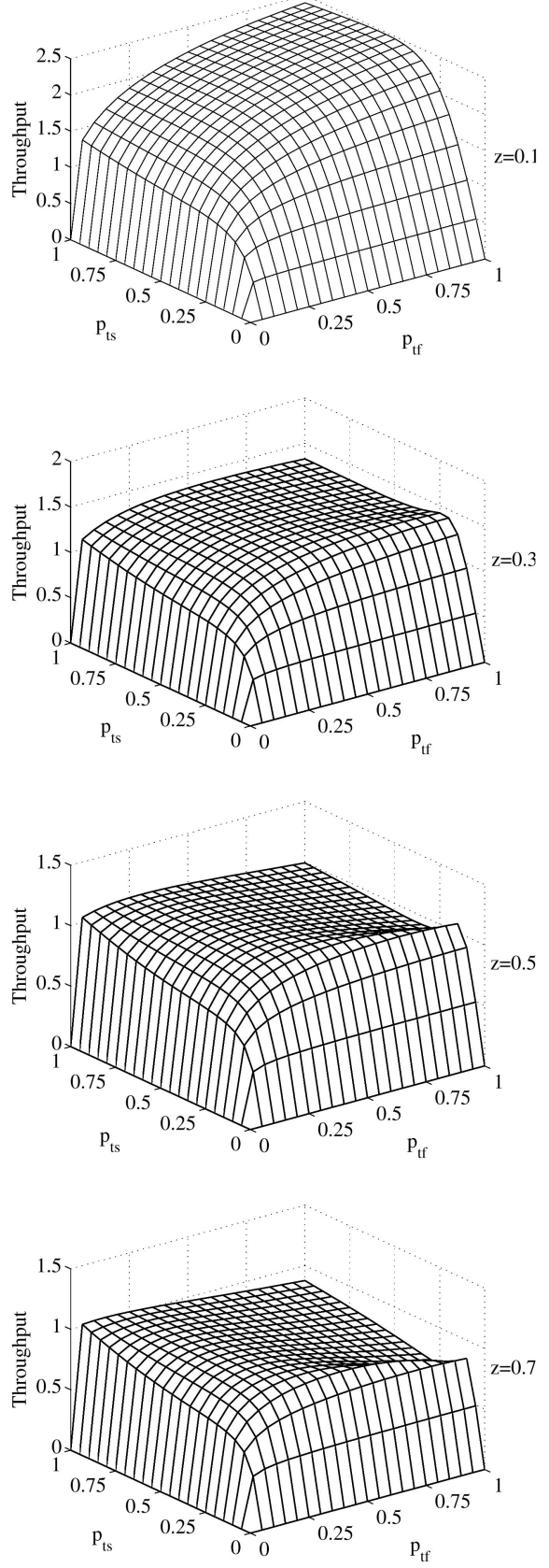

Fig. 14. The throughput $(S)$ under the GDP protocol in a system with fading. Ten nodes are distributed according to the disk model with $z=0.1, z=0.3, z=0.5$, and $z=0.7$.

In this paper, we focused on the design considerations of MAC protocols for networks of spatially distributed nodes with such capability. It is known that standard backoff mechanisms can lead to unfairness when nodes are spatially distributed. We demonstrated that with MPR capability, these mechanisms can also lead to significant throughput loss. To deal with this effect, we presented and analyzed an alternative backoff mechanism whereby nodes increase their transmission probability after a failed transmission and decrease it after a success. Our analysis shows that in most cases, the Alternative Model outperforms the Standard Model in terms of both throughput and fairness. Hence, new MAC protocols have to be designed for MPR networks and these protocols have to divert from the traditional backoff mechanisms.

This work is one of the first attempts to understand the design considerations of distributed MAC protocols for networks with MPR capability. Hence, there are still many open problems to deal with. As mentioned above, since the objective of this work is mainly to provide insight regarding MAC for networks with MPR capability, the studied protocols do not provide a complete solution. We intend to develop more elaborate backoff mechanisms that will utilize feedback from the receiver more effectively or will estimate the number of contenting nodes (e.g., the work of [52] for the IEEE 802.11 case). These mechanisms should deal with issues such as power, rate, and congestion control that have not been considered in this work and should be evaluated using the performance measures such as throughput, delay, and fairness. Moreover, since under different settings, different values of the protocol's parameters (e.g., $p_{\mathrm{ts}}, p_{\mathrm{tf}}, w_{s}, w_{f}$ ) lead to optimal performance, we intend to develop distributed mechanisms that would tune these parameters to the optimal values.

Furthermore, coexistence of the new backoff mechanisms with the standard backoff mechanisms (e.g., the one used in IEEE 802.11) while MPR and single-capture systems operate in parallel requires careful consideration. Finally, in most realistic scenarios, a transmission can be received by more than one destination (i.e., by multiple receivers). This provides diversity and flexibility that can be exploited by the MAC protocol. On the other hand, a transmission can interfere with other transmissions sent to a few different receivers. Thus, designing an efficient MPR MAC protocol for a multihop setting is a challenging open problem.

\section{ACKNOWLEDGMENTS}

This work was supported by US National Science Foundation (NSF) ITR grant CCR-0325401, NSF grants CNS-0915988 and CNS-0916263, ONR grant N000140610064, a grant from Draper laboratory, and a Marie Curie International Fellowship within the Sixth European Community Framework Programme. A preliminary verion of this paper was presented at IEEE INFOCOM, April 2008 [9].

\section{REFERENCES}

[1] N. Abramson, "The Throughput of Packet Broadcasting Channels," IEEE Trans. Comm., vol. COM-25, no. 1, pp. 117-128, Jan. 1977.

[2] J. Arnbak and W. Blitterswijk, "Capacity of Slotted ALOHA in Rayleigh-Fading Channels," IEEE J. Selected Areas in Comm., vol. SAC-5, no. 2, pp. 261-269, Feb. 1987.

[3] B. Bensaou, Y. Wang, and C. Ko, "Fair Medium Access in 802.11 Based Wireless Ad-Hoc Networks," Proc. ACM MobiHoc, Aug. 2000 .

[4] V. Bharghavan, A. Demers, S. Shenker, and L. Zhang, "MACAW: A Media Access Protocol for Wireless LAN's," Proc. ACM SIGCOMM, Aug. 1994.

[5] G. Bianchi, "Performance Analysis of the IEEE 802.11 Distributed Coordination Function," IEEE J. Selected Areas in Comm., vol. 18, no. 3, pp. 535-547, Mar. 2000.

[6] M. Carvalho and J.J. Garcia-Luna-Aceves, "A Scalable Model for Channel Access Protocols in Multihop Ad Hoc Networks," Proc. ACM MobiCom, Sept. 2004.

[7] P. Casari, M. Levorato, and M. Zorzi, "On the Implications of Layered Spacetime Multiuser Detection on the Design of MAC Protocols for Ad Hoc Networks," Proc. IEEE Int'l Symp. Personal, Indoor, and Mobile Radio Comm. (PIMRC '05), Sept. 2005. 
[8] G. Celik, “Distributed MAC Protocol for Networks with Multipacket Reception Capability and Spatially Distributed Nodes," master's thesis, MIT, http://web.mit.edu/gcelik/ MSThesis/Thesis.pdf, 2007.

[9] G. Celik, G. Zussman, W. Khan, and E. Modiano, "MAC for Networks with Multipacket Reception Capability and Spatially Distributed Nodes," Proc. IEEE INFOCOM, Apr. 2008.

[10] D. Chan and T. Berger, "Performance and Cross-Layer Design of CSMA for Wireless Networks with Multipacket Reception," Proc. IEEE Asilomar, Nov. 2004.

[11] I. Chlamtac and A. Farago, "An Optimal Channel Access Protocol with Multiple Reception Capacity," IEEE Trans. Computers, vol. 43, no. 4, pp. 480-484, Apr. 1994.

[12] I. Cidon, H. Kodesh, and M. Sidi, "Erasure, Capture and Random Power Level Selection in Multi-Access Systems," IEEE Trans. Comm., vol. 36, no. 3, pp. 263-271, Mar. 1988.

[13] M. Coupechoux, T. Lestable, C. Bonnet, and V. Kumar "Throughput of the Multi-Hop Slotted Aloha with Multi-Packet Reception," Proc. Conf. Wireless On-Demand Network Systems (WONS '04), pp. 301-314, 2004.

[14] J. del Prado Pavon, S. Shankar N, V. Gaddam, K. Challapali, and C.-T. Chou, "The MBOA-WiMedia Specification for Ultra Wideband Distributed Networks," IEEE Comm. Magazine, vol. 44, no. 6 , pp. 128-134, June 2006.

[15] M.-G. Di Benedetto, L. De Nardis, M. Junk, and G. Giancola, "(UWB $)^{2}$ : Uncoordinated, Wireless, Baseborn, Medium Access Control for UWB Communication Networks," ACM/Springer Mobile Networks and Applications, vol. 10, no. 5, pp. 663-674, Oct. 2005.

[16] A. Dua, "Random Access with Multi-Packet Reception," IEEE Trans. Wireless Comm., vol. 7, no. 6, pp. 2280-2288, June 2008.

[17] J.J. Garcia-Luna-Aceves, H. Sadjadpour, and Z. Wang, "Challenges: Towards Truly Scalable Ad Hoc Networks," Proc. ACM MobiCom, Sept. 2007.

[18] M. Garetto, T. Salonidis, and E. Knightly, "Modeling Per-Flow Throughput and Capturing Starvation in CSMA Multi-Hop Wireless Networks," Proc. IEEE INFOCOM, Apr. 2006.

[19] R. Gau and K. Chen, "Predictive Multicast Polling for Wireless Networks with Multipacket Reception and Queuing," IEEE Trans. Mobile Computing, vol. 5, no. 6, pp. 725-737, June 2006.

[20] S. Ghez, S. Verdu, and S. Schwartz, "Stability Properties of Slotted Aloha with Multipacket Reception Capability," IEEE Trans. Automatic Control, vol. 33, no. 7, pp. 640-649, July 1988.

[21] S. Ghez, S. Verdu, and S. Schwartz, "Optimal Decentralized Control in Random-Access Multipacket Channel," IEEE Trans. Automatic Control, vol. 34, no. 11, pp. 1153-1163, Nov. 1989.

[22] P. Gupta and P. Kumar, "The Capacity of Wireless Networks," IEEE Trans. Information Theory, vol. 46, no. 2, pp. 388-404, Mar. 2000.

[23] Z. Hadzi-Velkov and B. Spasenovski, "Capture Effect in IEEE 802.11 Basic Service Area under Influence of Rayleigh Fading and Near Far Effect," Proc. IEEE Int'l Symp. Personal, Indoor and Mobile Radio Comm. (PIMRC '02), Sept. 2002

[24] Z. Hadzi-Velkov and B. Spasenovski, "On the Capacity of IEEE 802.11 DCF with Capture in Multipath-Faded Channels," Int'l J. Wireless Information Networks, vol. 9, no. 3, pp. 191-199, July 2002.

[25] Z. Hadzi-Velkov and B. Spasenovski, "Capture Effect with Diversity in IEEE 802.11b DCF," Proc. IEEE Int'l Symp. Computers and Comm. (ISCC '03), June 2003.

[26] B. Hajek, A. Krishna, and R. LaMaire, "On the Capture Probability for a Large Number of Stations," IEEE Trans. Comm., vol. 45, no. 2, pp. 254-260, Feb. 1997.

[27] A. Krishna and R. LaMaire, "A Comparison of Radio Capture Models and Their Effect on Wireless LAN Protocols," Proc. IEEE Int'l Conf. Universal Personal Comm. (ICUPC '94), Sept. 1994.

[28] F. Kuperus and J. Arnbak, "Packet Radio in a Rayleigh Channel," Electronics Letters, vol. 18, no. 12, pp. 506-507, 1982.

[29] R. LaMaire, A. Krishna, and M. Zorzi, "On the Randomization of Transmitter Power Levels to Increase Throughput in Multiple Access Radio Systems," ACM/Baltzer Wireless Networks, vol. 4, no. 3, pp. 263-277, Mar. 1998.

[30] C. Lau and C. Leung, "Capture Models for Model Packet Radio Networks," IEEE Trans. Comm., vol. 40, no. 5, pp. 917-925, May 1992.

[31] N. Likhanov, E. Plotnik, Y. Shavitt, M. Sidi, and B. Tsybakov, "Random Access Algorithms with Multiple Reception Capability and N-Ary Feedback Channel," Problemy Peredachi Informatsii, vol. 29, no. 1, pp. 82-91, 1993.
[32] J. Linnartz, Narrowband Land-Mobile Radio Networks. Artech House, Inc., 1993.

[33] W. Luo and A. Ephremides, "Power Levels and Packet Lengths in Random Multiple Access," IEEE Trans. Information Theory, vol. 48, no. 1, pp. 46-58, Jan. 2002.

[34] W. Luo and A. Ephremides, "Power Levels and Packet Lengths in Random Multiple Access with Multiple-Packet Reception Capability," IEEE Trans. Information Theory, vol. 52, no. 2, pp. 414-420, Feb. 2006.

[35] A. MacKenzie and S. Wicker, "Stability of Multipacket Slotted Aloha with Selfish Users and Perfect Information," Proc. IEEE INFOCOM, Apr. 2003.

[36] M. Manshaei, G. Cantieni, C. Barakat, and T. Turletti, "Performance Analysis of the IEEE 802.11 MAC and Physical Layer Protocol," Proc. IEEE Symp. World of Wireless, Mobile and Multimedia Networks (WoWMoM '05), 2005.

[37] G. Mergen and L. Tong, "Receiver Controlled Medium Access in Multihop Ad Hoc Networks with Multipacket Reception," Proc. IEEE Military Comm. Conf. (MILCOM '01), Oct. 2001.

[38] G. Mergen and L. Tong, "Random Scheduling Medium Access for Wireless Ad Hoc Networks," Proc. IEEE Military Comm. Conf. (MILCOM '02), Oct. 2002.

[39] R. Merz, J. Widmer, J.-Y. Le Boudec, and B. Radunovic, "A Joint PHY/MAC Architecture for Low-Radiated Power TH-UWB Wireless Ad-Hoc Networks," Wireless Comm. and Mobile Computing, vol. 5, no. 5, pp. 567-580, Aug. 2005.

[40] J. Metzner, "On Improving Utilization in ALOHA Networks," IEEE Trans. Comm., vol. COM-24, no. 4, pp. 447-448, Apr. 1976.

[41] T. Moscibroda, Y. Oswald, and R. Wattenhofer, "How Optimal Are Wireless Scheduling Protocols?" Proc. IEEE INFOCOM, 2007.

[42] T. Nandagopal, T. Kim, X. Gao, and V. Bhargavan, "Achieving MAC Layer Fairness in Wireless Packet Networks," Proc. ACM MobiCom, Aug. 2000.

[43] G. Nguyen, A. Ephremides, and J. Wieselthier, "Comments on 'Capture and Retransmission Control in Mobile Radio'," IEEE J. Selected Areas in Comm., vol. 24, no. 12, pp. 2340-2341, Dec. 2006.

[44] G. Nguyen, J. Wieselthier, and A. Ephremides, "Accurate Capture Models and Their Impact on Random Access in Multiple-Destination Networks," Proc. IEEE Military Comm. Conf. (MILCOM '06), Oct. 2006.

[45] A. Nyandoro, L. Libman, and M. Hassan, "Service Differentiation Using the Capture Effect in 802.11 Wireless LANs," IEEE Trans. Wireless Comm., vol. 6, no. 8, pp. 2961-2971, Aug. 2007.

[46] M.C.H. Peh, S. Hanly, and P. Whiting, "Random Access with Multipacket Reception over Fading Channels," Proc. Australian Comm. Theory Workshop, Feb. 2003.

[47] M. Realp and A. Perez-Neira, "PHY-MAC Dialogue with MultiPacket Reception," Proc. ETSI Workshop Broadband Wireless Ad-Hoc Networks and Services, Sept. 2002.

[48] L. Roberts, "ALOHA Packet System with and without Slots and Capture," SIGCOMM Computer Comm. Rev., vol. 5, no. 2, pp. 28-42, 1975.

[49] R. Rom and M. Sidi, Multiple Access Protocols: Performance and Analysis. Springer-Verlag 1990.

[50] N. Shacham, "Throughput-Delay Performance of Packet-Switching Multiple Access Channel with Power Capture," Performance Evaluation, vol. 4, no. 3, pp. 153-170, Aug. 1984.

[51] H. Shekhar and M. Ingram, "Single and Multiple Packet Reception in a Random Access OFDMA System," Proc. IEEE Symp. Wireless Personal Multimedia Comm. (WPMC '07), Dec. 2007.

[52] A. Toledo, T. Vercauteren, and X. Wang, "Adaptive Optimization of IEEE 802.11 DCF Based on Bayesian Estimation of the Number of Competing Terminals," IEEE Trans. Mobile Computing, vol. 5, no. 9, pp. 1283-1296, Sept. 2006.

[53] L. Tong, Q. Zhao, and G. Mergen, "Multipacket Reception in Random Access Wireless Networks: From Signal Processing to Optimal Medium Access Control," IEEE Comm. Magazine, vol. 39, no. 11, pp. 108-112, Nov. 2001.

[54] X. Wang and J.J. Garcia-Luna-Aceves, "Embracing Interference in Ad Hoc Networks Using Joint Routing and Scheduling with Multiple Packet Reception," Proc. IEEE INFOCOM, Apr. 2008.

[55] Z. Wang, H. Sadjadpour, and J.J. Garcia-Luna-Aceves, "The Capacity and Energy Efficiency of Wireless Ad Hoc Networks with Multi-Packet Reception," Proc. ACM MobiHoc, May 2008.

[56] Q. Zhao and L. Tong, "A Multiqueue Service Room MAC Protocol for Wireless Networks with Multipacket Reception," IEEE/ACM Trans. Networking, vol. 11, no. 1, pp. 125-137, Feb. 2003. 
[57] Q. Zhao and L. Tong, “A Dynamic Queue Protocol for Multiaccess Wireless Networks with Multipacket Reception," IEEE Trans. Wireless Comm., vol. 3, no. 6, pp. 2221-2231, Nov. 2004.

[58] P. Zheng, Y. Zhang, and S. Liew, "Multipacket Reception in Wireless Local Area Networks," Proc. IEEE Int'l Conf. Comm. (ICC '06), June 2006.

[59] M. Zorzi and R. Rao, "Capture and Retransmission Control in Mobile Radio," IEEE J. Selected Areas in Comm., vol. 12, no. 8, pp. 1289-1298, Oct. 1994

[60] M. Zorzi and R. Rao, "Reply to 'Comments on 'Capture and Retransmission Control in Mobile Radio",", IEEE J. Selected Areas in Comm., vol. 24, no. 12, pp. 2341-2342, Dec. 2006.

[61] M. Zorzi, "Mobile Radio Slotted ALOHA with Capture, Diversity and Retransmission Control in the Presence of Shadowing," ACM/ Baltzer Wireless Networks, vol. 4, no. 5, pp. 379-388, 1998.

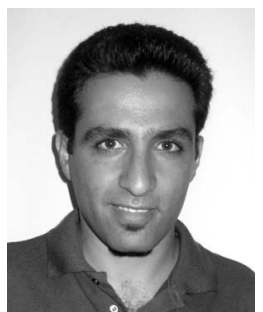

Guner D. Celik received the BS degree in electrical and electronics engineering from the Middle East Technical University (METU), Ankara, Turkey, in 2005, and the SM degree in electrical engineering and computer science (EECS) from the Massachusetts Institute of Technology (MIT) in 2007. He is currently working toward the $\mathrm{PhD}$ degree at the Electrical Engineering and Computer Science Department, MIT. In the summer of 2007, he joined Microsoft Research Systems and Networking Group at Cambridge UK as a research intern. His research interests are in the area of wireless communication networks. In particular, he is interested in the design and analysis of algorithms and protocols for both mobile and static wireless networks. He received the METU President's Award in 2002, the second highest ranking in the Faculty of Engineering, and the Golden Graduation Project Award in METU in 2005. He is a student member of the IEEE.

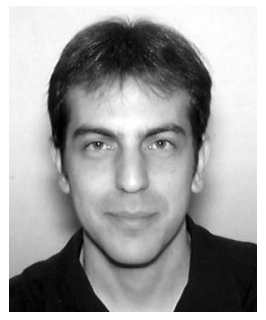

Gil Zussman received the BSc degree in industrial engineering and management and the BA degree in economics from the TechnionIsrael Institute of Technology in 1995 (both summa cum laude), the MSc degree (summa cum laude) in operations research from Tel-Aviv University in 1999, and the PhD degree in electrical engineering from the Technion in 2004. Between 1995 and 1998, he served as an engineer in the Israel Defense Forces, and between 2004 and 2007, he was a postdoctoral associate at the Massachusetts Institute of Technology. He is currently an assistant professor in the Department of Electrical Engineering, Columbia University. His research interests are in the area of wireless networks. He received the Marie Curie Outgoing International Fellowship, the Fulbright Fellowship, the IFIP Networking 2002 Best Student Paper Award, and the OPNETWORK 2002 and ACM SIGMETRICS/IFIP Performance 2006 Best Paper Awards. In 2009, he received the DTRA Young Investigator Award, and was a member of a group that won the first place in the Vodafone Foundation Wireless Innovation Project competition. $\mathrm{He}$ is a senior member of the IEEE.

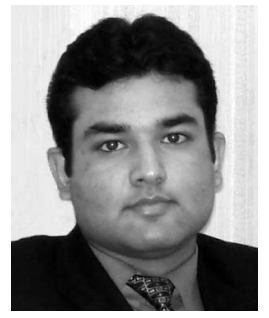

Wajahat F. Khan received the BS degree in electrical science and engineering, and the BS degree in mathematics with computer science and the MEng degree in electrical engineering and computer science from the Massachusetts Institute of Technology (MIT) in 2007 and 2008, respectively. His master's research focused on novel autonomous routing protocols for networks with wide-spread failures. $\mathrm{He}$ is currently working for Texas Instruments, Dallas, in the Open Multimedia Application Platform group. His research interests are in communications, networking, and computing. $\mathrm{He}$ is a member of the IEEE.

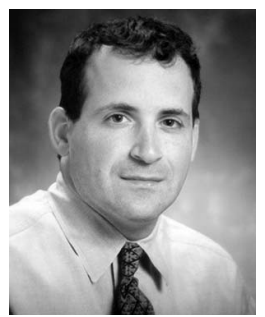

Eytan Modiano received the BS degree in electrical engineering and computer science from the University of Connecticut at Storrs in 1986, and the MS and $\mathrm{PhD}$ degrees in electrical engineering from the University of Maryland, College Park, in 1989 and 1992, respectively. He was a US Naval Research Laboratory fellow between 1987 and 1992 and a National Research Council postdoctoral fellow during 19921993. Between 1993 and 1999, he was with the Massachusetts Institute of Technology (MIT) Lincoln Laboratory, where he was the project leader for the MIT Lincoln Laboratory's Next Generation Internet (NGI) project. Since 1999, he has been on the faculty at MIT, where he is presently an associate professor. His research is on communication networks and protocols with emphasis on satellite, wireless, and optical networks. He is currently an associate editor for the IEEE/ACM Transactions on Networking and the International Journal of Satellite Communications and Networks. He had served as an associate editor for the IEEE Transactions on Information Theory (communication networks) and as a guest editor for the IEEE JSAC special issue on WDM network architectures, the Computer Networks Journal special issue on broadband Internet access, the Journal of Communications and Networks special issue on wireless ad-hoc networks, and the IEEE Journal of Lightwave Technology special issue on optical networks. He was the technical program cochair for Wiopt 2006, IEEE INFOCOM 2007, and ACM MobiHoc 2007. He is a senior member of the IEEE.

$\triangleright$ For more information on this or any other computing topic, please visit our Digital Library at www.computer.org/publications/dlib. 